\title{
Public health policy, evidence, and causation. Lessons from the studies on obesity
}

\author{
Federica Russo \\ Philosophy, University of Kent
}

Draft of October 20, 2010

\begin{abstract}
The paper addresses the question of how different types of evidence ought to inform public health policy. By analysing causal studies on obesity, the paper draws lessons about the different roles that different types of evidence play in setting up public health policies. More specifically, it is argued that 'difference-making' evidence supports considerations about 'what works for whom in what circumstances', and that 'mechanistic evidence' provides information about the 'causal pathways' to intervene upon.
\end{abstract}

Keywords: Causation; Disease Causation; Evidence; Evidence-based Public Health; Obesity; Public Health Policy.

\section{The fat generation in the era of 'evidence'}

Health is a concern for particular individuals as well as for policy makers. My interest in this paper goes to the worries of policy makers, who are committed to find effective ways to improve health of populations. No wonder public health policy is utterly concerned with questions about evidence, that is with questions about the very basis of public health interventions. There are of course a plethora of issues looming here; in this paper I shall restrict the discussion to the roles that different types of evidence ought to play in setting up public health policies. Studies on obesity are exemplar, as we shall see, in exhibiting the complexity of the quarrels about policy, evidence and causation; at the same time, these studies also provide fruitful insights to answer questions about evidence and causation in the public health policy debate. Let me then open the paper with an outlook of the studies on obesity.

Obesity is a chronic disease. The prevalence of obesity - i.e., its percentage with the respect to the total population - is increasing in many countries and at different ages. Interest in obesity is not in its being a new disease, as obese people existed in the past too, but rather in the fact that the number of obese people is growing, and very fast. 
In more and more Member States of the European Union, over $20 \%$ of the adult population is obese, over $50 \%$ of the adult population is overweight, and up to $20 \%$ of children are overweight. ${ }^{1}$

Figures show that obesity is now an epidemic. Evidence collected in numerous studies shows that diseases related to excess adipose tissue - e.g., cardiovascular diseases - have drastically increased. Moreover, those diseases are amongst the most significant factors of morbidity and mortality worldwide; the contention of those studies is that, ultimately, prevalence in obesity and its associated comorbidites is likely to continue to increase in the near future.

The question easily arises: how to explain such an increase in the percentage of obese people? There are, broadly speaking, two main factors: biological and genetic factors on the one hand, and nutrition and lifestyle on the other hand.

These two broad categories of factors correspond to two perspectives on the disease: biological and socio-economic. Not only there exist different perspectives on the disease, but also different ways in which different people involvedoverweight people, health professional, policy makers-perceive the problem (see e.g. Greener et al. (2010); Galea et al. (2010)).

Also, in understanding obesity, there seem to be two distinct albeit related questions at stake: (i) what are the causes of obesity and (ii) what are the causes of the increase of prevalence obesity. Whilst the first question is concerned with having a thorough understanding of the disease, it is in fact the second that is most relevant for public health purposes.

It may be argued that the action taken in response to the second question above, also depends on the answers given to the first question. In other words, the better our understanding of the disease and of its development, the better our actions to reduce its burden on population. Nevertheless, this is easier said than done. It is in fact the complexity of disease causation in this case that makes interventions so hard to put in place. Research suggests that most human obesity probably reflects complex interactions between genetic, environmental, and social factors often mediated through nongenetically derived changes in metabolism. The phenomenon is so complex that some are suggesting computer simulation through the so-called 'agent-based models' to get a better grip on it (see e.g. Galea et al. (2010))

An interesting aspect of obesity is that both 'health' and 'economic' considerations motivate public health intervention. I mentioned earlier consequences on health such as cardiovascular problems. But there are consequences other than health: standard seat width increased, office furniture, revolving doors, average passengers weight implications for fuel used by airlines, all equipment in hospitals (bed, wheelchairs, operating tables, etc). We thus want to reduce obesity rates for its effects on health, but not only.

There are already a number of public health interventions in place to reduce obesity. For instance, the MEND programme ${ }^{2}$ proved to be successful in reducing obesity. Established in 2004, MEND aims to teach children and their

\footnotetext{
${ }^{1}$ This data refers to statistics published on open access websites and data bases of the European Commission, the UK National Health Services, and the World Health Organisation, all accessed in March 2010 (http://www.hopeproject.eu/index.php?nav_id=; http://www.ic.nhs.uk/statistics-and-data-collections/health-and-lifestyles/ obesity/statistics-on-obesity-physical-activity-and-diet-england-2010; http: //www.who.int/mediacentre/factsheets/fs311/en/index.html)

${ }^{2}$ Information about the programme, the services, the follow-up, related scientific publications, etc. can be found at the sable URL=http://www. mendprogramme.org/home.
} 
families how to live healthier. By targeting children in the age ranges of $2-4,5-$ 7, and 7-13, MEND programmes also target the parents of obese children thus aiming to positively change their and the children's habits about nutrition and lifestyle. Likewise, there are around a number of interventions and campaigns sponsored by different national health services and by the European Commission in order to induce changes in dietary habits and consequently to reduce obesity.

Ultimately, the difficulty is to decide what policies will be effective, and for whom. In other words, two issues are at stake here:

(i) What factors - i.e., biological/genetic or social - to intervene upon to reduce obesity.

(ii) Who should take part in public health policies to reduce obesity what level to target the intervention: children, adults, poor people, ...

Thus, the question arises: what evidence ought to inform public health policy? The answer I will give is that two types of evidence broadly conceived ought to inform public health policy: difference-making and mechanistic. Whilst the former supports considerations about 'what works for whom and in what circumstances', the latter provides information about the 'causal pathways' to intervene upon. The argument will run in three steps.

Section 2 states the importance of epidemiology for public health because of its population-level perspective on disease causation. It is then argued that in epidemiology (and in medicine more generally) causal assessment requires evidence of two types: difference-making and mechanistic. Arguments in favour of this thesis can be also found in Russo (2009, 2011); Russo and Williamson (2007, 2011).

Section 3 praises the efforts of public health practitioners to address questions of evidence. It is argued that although they work towards developing criteria for evidence assessment, the most crucial question, that is what evidence is needed for public health policy, is by and large left unanswered.

Section 4 provocatively pleads for causally-based public health on the grounds that a better understanding of disease causation will result in better actions and decision. Thus, if we accept the idea that disease causation requires two types of evidence - difference-making and mechanistic - so does public health policy. Difference-making evidence, mainly in the form of risks coming from descriptive epidemiology, supports considerations about 'what works for whom in what circumstances'. Mechanistic evidence, mainly coming from analytic epidemiology, cashes out the 'causal pathways' upon which we have to intervene. In sum, these different types of evidence serve different roles in deciding about public health policy.

\section{Public health and epidemiology}

Public health aims to preventing disease, prolonging life, and promoting health of populations. A first peculiar characteristic of public health is that actions to reach those aims are based on population-level analyses. Thus, public health is concerned with preventive rather than curative and individual-level interventions; individual-level measures are in fact a concern of medicine (and, within the broad evidence-based perspective, a concern of evidence-based medicine). 
A second characteristic of public health, as practitioners notice, is its interdisciplinarity. The decisions about what actions to undertake rely on findings in other areas, such as epidemiology, biostatistics, behavioural sciences, health economics and and health care management. Given the population-level dimension of public health, I am most concerned with the importance of epidemiology, for reasons that will become clear throughout the discussion.

Epidemiology studies how the distributions of diseases and their biological and socio-economic determinants vary within a population and across different populations. This happens in two, typically subsequent, kinds of analyses: descriptive and analytic epidemiology. On the one hand, descriptive epidemiology seeks to answer questions about 'who' in the population is affected by 'what' disease and under what circumstances ('when' and 'where'). The main goal of descriptive epidemiology is thus to work out risks of disease and exposure for a given population. On the other hand, analytic epidemiology seeks to answer questions of 'how' the disease operates and 'why' it develops and spreads. The main focus of analytic epidemiology are measures of associations from which to draw inferences about the causes of disease. Thus, analytic epidemiology is certainly more 'causally-oriented' than descriptive epidemiology. The causal character of analytic epidemiology is not always made explicit (see e.g. Porta (2008)). Nevertheless, in asking how and why questions, analytic epidemiology clearly goes beyond the mere measurement of associations and evaluates mechanistic explanatory hypotheses.

Here is an example. Constantin et al. (2010) are interested in measuring associations between leptin G-2548A and leptin receptor Q223R gene polymorphisms; yet, the motivation is to test mechanistic hypotheses. In the quote below I have highlighted in bold in the mechanistic hypotheses that the epidemiologists aim to test, and I have italicised the corresponding associations that they measure in order to test it.

Obesity arises from a complex interaction between genetic variance, environment, and lifestyle changes. Obesity is one of the most challenging health problems of the last century with a tremendous increase in the prevalence, considered to be an important risk factor for type 2 diabetes and cardiovascular diseases.

Leptin, the obese (ob) gene product acts to reduce food intake and to increase energy expenditure, and plays an important role both in the development of obesity and in insulin secretion.

Leptin exerts its pleiotropic actions directly through distinct receptors (Ob-R) encoded by the diabetes $(d b)$ gene. In humans, $L E P$ and $L E P R$ have been mapped to $7 \mathrm{q} 31.3$ and $1 \mathrm{p} 31$, respectively. The long form of leptin receptor OB-Rb is thought to be essential in leptins weight-reducing effects through the hypothalamus and.

Serum leptin level is significantly increased in obese persons and is proportionally with body adiposity. In type 2 diabetes leptin levels have been reported to be either unchanged or reduced.

The leptin $(L E P)$ and leptin receptor $(L E P R)$ genes have been investigated in the search for gene variants potentially related to the pathophysiology of obesity, diabetes and its associated complica- 
tions. A leptin gene SNP consisting in G to A substitution at nucleotide (nt) -2548 upstream of the ATG start site in the LEP gene promoter, LEP G-2548A, has been associated with adipocytes increased leptin production and secretion. Interestingly, as for $L E P R$ gene polymorphism, the $A$ to $G$ transition in exon 6 at nt 668 from the start codon 223 (Q223R) was associated with impaired leptinbinding activity. The LEPR Q223R polymorphism has been associated with body mass index (BMI), fat mass, leptin levels, and systolic and diastolic blood pressure. There are few studies dealing with the association between $L E P$ G-2548A and LEPR Q223R polymorphisms, type 2 diabetes, and obesity status. Thus, in the Chinese population, LEP -G2548A showed a positive correlation with incidence of type 2 diabetes. Recently, it was reported that $L E P R$ Q223R polymorphism has been associated with impaired glucose tolerance and conversion to type 2 diabetes, and insulin resistance. The aim of this study was to investigate whether two common single nucleotide polymorphisms (SNPs) in the leptin (LEP G-2548A) gene and its receptor (LEPR Q223R) gene are related to obesity in a sample of urban Romanian population. Also, the influence of the LEP G-2548A and the LEPR Q223R polymorphisms on the variability of metabolic phenotypes (anthropometric variables, glucose, insulin and leptin concentration, and lipid profile) was investigated.

Although analytic epidemiology does not state in such clear terms that the stake is the evaluation of mechanistic hypotheses, it is hard to think of answers to 'why' and 'how' questions that are not causal and mechanistic. In addressing the 'why' question, analytic epidemiology must be looking for the causes of disease, rather than mere associations between risk factors or exposure and disease. To say it with Wesley Salmon, successful explanations are those that put "the cause into because" (Salmon, 1984). As for the 'how' question, here a satisfactory answer must consider the functioning of, or the modes of organisation and interaction within, the phenomenon being analysed. This is the core idea behind mechanistic explanations, which is defended, in slightly different ways both by scholars in the 'mainstream' literature on mechanisms (see, e.g., Bechtel and Abrahamsen (2005)) and by scholars interested in the explanatory import of models in social science (see, e.g., Mouchart and Russo (2010)).

As mentioned above, epidemiology is perhaps the main source of information for public health. Yet, it is worth noting that the public health dimension of epidemiology is a matter of controversy amongst epidemiologists themselves. Although many share the view that epidemiology underpins public health, there is still a wide gap between public health practice and academic epidemiology (on this point see for instance Bophal (1997, 1999) and references therein).

Research in epidemiology is arguably driven by two distinct but nonetheless complementary goals: (i) to understand and learn about disease and (ii) to take action in order to reduce the burden of disease at the population level. This second, more controversial goal will be thoroughly discussed in $\S 3$. Let us focus now on the first one. Understanding and learning about disease consists in drawing conclusions from evidence, more particularly conclusions about disease causation. Therefore, epidemiology brings to the fore questions about what evidence is needed to draw conclusions about disease causation. 
We have seen in $\S 1$ that epidemiological studies on obesity aim to reach a comprehensive understanding of the disease. This is done by seeking answers to questions about 'who-what-when-where', identifying the relevant risk factors, viz. by making considerations about difference-making, and by seeking answers to the 'how-why' questions which are about mechanisms. This thesis - that causal assessment typically needs difference-making and mechanistic evidencehas been defended for the health sciences in general and for causal inference in epidemiology and autopsy in particular by Russo $(2009,2011)$ and by Russo and Williamson (2007, 2011). I will not reiterate the arguments already given in favour of the thesis. Instead, in $\S 3$ I defend the idea that the two types of evidence are also needed for public health purposes.

Let me give a scent of what these two types of evidence amount to. Simply put, difference-making evidence is evidence that a putative causal factor 'makes a difference' to the putative effect. In other words, difference-making evidence helps deciding whether the putative causal factor is relevant for the occurrence of the putative effect. This type of evidence can be cashed out in different forms: probabilistic, statistical, or counterfactual relations. Difference-making evidence is especially needed for description and for prediction of disease causation. Mechanistic evidence is about a plausible or confirmed enough mechanisms that are meant, in turn, to support results of difference-making. Mechanistic evidence is especially needed for explanation and control. The characterisation hereby offered is very general and the interested reader may want to have a look at Gillies (2010) and Illari (2010) for a critical discussion.

\section{$3 \quad$ Evidence-based public health}

Consider now the second goal of epidemiology mentioned above, i.e. taking action in order to reduce the burden of disease. Although some epidemiologists do think that this is their concern too, in the last years public health has worked towards establishing itself as an autonomous scientific domain (with respect to the many other disciplines that inform public health policy) within the so-called evidence-based movement.

One motivation to embrace the evidence-based framework is to provide decisions and actions with a stronger basis. For instance, Brownson et al. (1999, p.87) notice that

[i]deally, public health practitioners always incorporate scientific evidence in making management decisions, developing policies, and implementing programs. However, in reality, these decisions often are based on short-term demands rather than long-term study, and policies and programs are developed frequently around anecdotal evidence.

Consequently, the advent of evidence-based public health is certainly welcome. But, at the same time, evidence-based public health (EBPH) brings to the fore specific questions about the basis upon which policy ought to be based, and, moreover, it inherits from epidemiology crucial questions about evidence and causal assessment. This can be extracted from the way EBPH theorists define their own discipline. We read, for instance: 
Evidence-based public health is defined as the development, implementation, and evaluation of effective programs and policies in public health through application of principles of scientific reasoning, including systematic uses of data and information systems, and appropriate use of behavioral science theory and program. (Brownson et al., 2003)

Evidence-based public health is the process involved in providing the best available evidence to influence decisions about the effectiveness of policies and interventions and secure improvements in health and reductions in health inequalities. (Killoran and Kelly, 2010, p. xxii)

From the quotes above it emerges that the main concern of EBPH is to provide a solid, scientific basis for the decisions and actions taken to reduce the burden of disease. Multiple sources of evidence are said to be necessary to this end.

Indeed, the focus on evidence concerns many aspects (Guyatt and Drummond, 2002): what evidence one's practice or policy is based upon, the soundness of evidence, the strength of inference permitted by evidence, etc. Put it in more general terms, what is key is the assessment of evidence. ${ }^{3}$

There is a broad consensus that assessing scientific evidence involves evaluating peer review publications by conducting meta-analyses in order to minimise problems of bias and to decide about the quality and generalisability of studies. Evidence assessment is also usually taken to involve quantitative risk assessment, economic evaluation of the prospective interventions, and consultations with expert panels. Brownson et al. (2003, ch. 2), a classic in EBPH, explicitly mention issues related to causality. They start with the usual caveat that causality is almost impossible to establish with certainty; they then discuss wellknown criteria and guidelines used in the health sciences such as Henle-Koch postulates and Bradford Hill's guidelines. They also review pioneering and influential methods put forward by a number of accounts of epidemiologists that overtly embraced a causalist perspective (most famously, Rothman's 'pie' and Susser's 'Chinese boxes'). However, according to Brownson et al. (2003), causal issues are eventually resolved by following Hill's guidelines. No more, no less.

It is worth emphasising that the worries of evidence-based practitioners concern the best evidence to license inference and action. However, in the discussions about how to assess what evidence is best, a clear statement about what is the evidence that undergoes assessment is missing. This is the point I am most concerned with. Even if we agree that Hill's guidelines provide a comprehensive enough list for causal assessment, we need to make clear what these guidelines help us assessing.

Granted, there is an effort in providing a positive, more specific account of what evidence is to undergo evaluation for public health purposes. Notably, some EBPH practitioners distinguish between Type I and Type II evidence. Type I evidence points to a particular health condition for which some preventable risk factors have been identified. Such evidence tells that something

\footnotetext{
${ }^{3}$ It goes without saying that, when discussing policy, questions about what ethical principles and values ought to guide interventions inevitably come up. Those are certainly important. However, I want to focus here on a theoretical issue, namely on the theoretical issue of what constitutes evidence for policy in public health contexts.
} 
must be done. Type II evidence points to specific interventions that proved to be relatively efficacious in order to improve a particular health condition. Such evidence tells that this particularly must be done. The evaluation of either type of evidence is done through systematic reviews of findings in well-conducted studies, especially with attention to their internal and external validity (Brownson et al., 1999). This brings us straight onto the next point about evidence assessment.

EBPH theorists, just like EBM theorists, oft appeal to the so-called 'evidence hierarchy' and claim that the best evidence comes from randomized clinical trials - to be sure, from systematic reviews of several RCTs. There are a number of arguments that may be developed against this claim or against the 'received' hierarchy altogether. I will not endorse this line of argument here, though. I just want to flag the issue and report that critical views come from the scientific community itself, not just the philosophical community. For instance, Rychetnik et al. (2004) admit that RCTs are important for causal assessment but make the point that the complexity of public health requires that RCTs be accompanied by other forms of causal assessment, e.g. observational studies using adequacy or plausibility design. Likewise, Glasziou et al. (2007) make the point that sound causal conclusions may be reached through observational methods too; consequently RCTs are mistakenly considered the gold standard of causal inference. However, as I said, I will not pursue this line argument here.

In spite of the valuable attempts to give evidence assessment the shape, the evidence hierarchy - just like the distinction between Type I and Type II evidence - leaves largely unanswered the question of what evidence is in fact needed for policy.

To be sure, this is a concern shared by theorists of EBPH too. Witness for instance Rychetnik et al. (2004, p. 538):

In the broadest sense, evidence can be defined as "facts or testimony in support of a conclusion, statement or belief" 4 . Such a generic definition is a useful starting point, but it is devoid of context and does not specify what counts as evidence, when, and for whom.

What evidence be needed for policy is likewise left unspecified in official documents of agencies such as the European Food Safety Authority (EFSA) ${ }^{5}$. Consider for instance the "Regulation (EC) No 1924/2006 of the European Parliament and of the Council of 20 December 2006 on nutrition and health claims made on foods" ${ }^{6}$. The first chapter of the document provides precise definitions of the terms that will be subsequently used, such as 'nutrient', 'health claim', 'reduction of disease risk claim' etc. Throughout the document we are repeatedly told that evaluation of foods is made on the basis of scientific evidence, and yet, evidence is never defined.

A charitable interpretation would be that what is meant by evidence in this context is exactly the evidence discussed in scientific publications of evidencebased public health theorists, as the ones mentioned above. If this is the case,

\footnotetext{
${ }^{4}$ Trumble W.R., Stevenson A. (eds), Shorter Oxford English dictionary on historical principles, Oxford: Oxford University Press, 2002.

${ }^{5}$ Information about EFSA, its goals, organisation, documents, campaign and events can be retrieved from its portal, URL=http://www.efsa.europa.eu/.

${ }^{6}$ The document may be accessed at the following stable URL=http://eur-lex.europa. eu/LexUriServ/LexUriServ. do?uri=0J : L : 2006 : 404:0009:0025: EN : PDF.
} 
I contend that Type I and Type II evidence nor the evidence hierarchy answer the question of what evidence is needed for public health in a satisfactory way. Moreover, what is left unanswered is what roles different types of evidence serve in making policy decisions. This, I shall examine next.

\section{Causally-based public health}

\subsection{Understanding and acting: causally-based scientific practices}

Let the following idea guide the arguments below. As a general rule, making good decisions and taking good actions depend on having a good understanding of the phenomenon or situation. To be more specific about public health policies, the better our understanding of the disease, the better too the interventions to reduce the burden of disease. Thus, if we accept the idea that understanding disease causation involves making considerations about different types of evidence (see §2), then those different types of evidence should feed the design of public health policies too.

Killoran and Kelly (2010, p. xxii-xxiii) make a list of what they take to be the key features of EBPH. Two of them, in particular, matter for the present discussion. One is "conceptual plausibility: an understanding of causal pathways defining the factors influencing health and the potential for intervention"; the other is "use of different types of evidence to determine what works for whom in what circumstances".

This patently brings upfront in the discussion of evidence-based public health causal questions of the type I mentioned at the end of $\S 2$, namely what kinds of evidence are typically involved in causal assessment. I argue below that mechanistic evidence cashes out Killoran and Kelly's 'causal pathways' and difference-making backs up their 'what works for whom in what circumstances'.

Thus, this section provocatively pleads for the idea that public health policy should be causally-based, not just evidence-based. Some may immediately rebut that causality is too strong a requirement for policy. Not only this is an area where a good grip on causal relations is rarely obtained; moreover, as a matter of fact, decisions and actions are normally based on sole knowledge of risk factors and their strength. To this it may be counterargued that to intervene on factors that are not the causes of disease would eventually turn out to be useless. Consequently, to ensure (as much as possible) that policies are effective, we have to intervene on the causes of disease, not on factors merely associated to the disease. Moreover, even if it is common practice to design public health policies on the mere basis of risks, this is bad practice notwithstanding their strength. I explain below why evidence of mechanisms, beside difference-making evidence cashed out in the form of risks, offers a more solid basis to public health policy.

Concerns about the relations between evidence and policy have been also raised by Nancy Cartwright. Cartwright (2009) argues that causal knowledge is valuable for policy and planning. In particular, she is interested in the connection between causal knowledge and the ability to predict results of manipulations or interventions. To this end, what is most relevant, according to Cartwright, is whether 'invariance' and 'modularity' are able to deliver causal relations, as many people believe. In fact, invariance (i.e., that model parameters exhibit 
some kind of stability across changes of environments or interventions) and modularity (i.e., that we can intervene on one factor to see its effects without affecting the whole system) are usually taken to be the fundamental characteristics that allow structural models (i.e., the models customarily used in economics and econometrics) to correctly represent causal relations.

Whilst I am concerned with the general problem of the types of evidence to establish whether a relation is causal, Cartwright, as I see it, is more particularly concerned with the assessment of difference-making evidence, cashed out in terms of invariance and modularity in structural models.

But Cartwright (2008) is also concerned with what claims are relevant for policy and, she argues, the relevant claims are causal claims. It is along those lines of reasoning that I will next make the case for the claim that the two types of evidence involved in causal assessment ought also to be involved in public health policy, because of the specific role each serves.

It is worth making clear that the normative claim is that difference-making and mechanistic evidence should explicitly enter EBPH guidelines, such as the ones of the European Food Safety Authority mentioned earlier. On the one hand, it is a matter of fact that theses two types of evidence are, one way or another, already used by EBPH practitioners, albeit just implicitly. On the other hand, it is also a matter of fact that these two types of evidence are not explicitly mentioned in various EBPH guidelines (textbooks or official documents of various agencies). Notice, though, that I am not claiming that no action is possible without detailed evidence of each of the two types. Rather, my point is that better action can be planned if those are explicitly taken into account, as much as possible and subject to their availability. There certainly are situations in which some decision must be taken even in absence of full evidence (most typically, in absence of good mechanistic evidence). It does not follow, though, that research to collect further (mechanistic) evidence is not needed anymore.

\subsection{Difference-making and mechanistic evidence}

In this section it is argued that public health policy needs back up coming from two types of evidence broadly conceived - difference-making and mechanisticeach serving a specific role in public health policy.

Difference-making evidence Public health policy needs information coming from difference-making evidence. Difference-making evidence may be in the form of e.g. statistical relevance relations, probabilistic dependencies, risks. This is typically provided by descriptive epidemiology, which answers questions about who, what, when, where.

Such information is helpful in deciding whether policy interventions have to target the whole population (e.g., in food labelling), or only subgroups (e.g., families as in the MEND) or individuals (e.g., in MEND there is also the possibility to report an obese child) that fall under certain categories. On a different level, difference-making evidence may also help in deciding whether it is more efficient to intervene on the social or biological factors of disease (or on both simultaneously).

Thus, the role of difference-making evidence is to back up considerations about what works for whom in what circumstances. Part of the job is to make 
it plausible that what worked for some population will also work in another one based, by and large, on arguments by analogy. ${ }^{7}$ Such information is collected, for instance, by local and national cancer registers or in obesity databases. ${ }^{8}$ There are also a number of research projects promoted by the Executive Agency for Health and Consumers. ${ }^{9}$ Although those projects are listed under policy actions, they are in fact highly exploratory, in that they are meant to provide relevant difference-making information for actions such as prevention in children or particular classes of professionals.

Mechanistic evidence Public health policy needs mechanistic evidence too; this is provided by analytic epidemiology, which is mainly concerned with explanatory causal hypotheses and answers questions about 'how' and 'why'. Recall, the goal of analytic epidemiology is to design studies to test hypotheses that come from descriptive epidemiology. The scientific literature stresses the use of measures of associations (e.g., relative risks and odds ratios) and hypothesis testing. However, arguably, this is just half of the story. In fact, measures of association and hypothesis testing need the underpin of mechanistic evidence in order to be explanatory (or otherwise they just restate difference-making evidence).

A good example is provided by the studies on obesity and type 2 diabetes, more particularly on the mechanisms regulating insulin resistance. Those investigations are motivated by descriptive studies that reveal neat correlations between increased numbers of overweight or obese people and numbers of people affected by diabetes. ${ }^{10}$

Thus, the role of mechanistic evidence is to provide information about the causal pathways upon which to intervene. Notice, though, the such pathways need not be sharply nor only biological. There is in fact substantial investigations on social determinants and health inequalities in general and for obesity in particular (see also below 'ecological' views of obesity). ${ }^{11}$

A number of remarks are in order.

First, although there is no 'conceptual' priority of one type of evidence over the other, it is true that different types of evidence may have unequal weights in assessing different hypotheses of disease causation. For instance, in addressing the question of what caused the increased obesity prevalence, difference-making evidence is more important for the 'fast-food hypothesis'. According to this hypothesis, the drastic changes in dietary habits, and in particular the increased consumption of greasy meals prepared in fast food restaurants explains the rapid

\footnotetext{
${ }^{7} \mathrm{I}$ say 'part of the job' and 'by and large' because this leads us straight into problems of external validity, which are far from being settled. For a novel and thought provoking account of external validity see Steel (2008); for a discussion of the insufficiency of statistics for external validity, see Cartwright (2010).

${ }^{8}$ See for instance the National Childhood Obesity Database 2005-2006 (http: //www.dh.gov.uk/en/Publicationsandstatistics/Publications/PublicationsStatistics/ DH_063565)

${ }^{9}$ See for instance http://ec.europa.eu/eahc/projects/database.html?prjno=2003305, or http: //ec. europa. eu/eahc/projects/database.html?prjno=2004313.

${ }^{10}$ See for instance http://ec.europa.eu/research/leaflets/diabetes/index_en.html. Here is a list of projects funded under the 5th and 6th Framework Programme: http: //cordis.europa.eu/lifescihealth/major/diabetes-eu-funding.htm.

${ }^{11}$ See for instance http://ec.europa.eu/health/social_determinants/policy/index_en. htm.
} 
increase of obesity in the last fifty years or so. In this case difference-making evidence - namely about 'who' eats 'what' and 'when'-is more important to pick out the right targets for intervention, as the mechanism is pretty obvious: a much easier access to food, which is in turn richer in fat than it used to be, makes people get fat very quickly. On the other hand, when investigating the genetic hypothesis, mechanistic evidence is the primary focus and difference-making evidence will play an auxiliary role. According to this hypothesis the main factor explaining obesity is some particular genetic make up that in turn regulates insulin resistance. Thus, what is most important is to work out the 'causal pathways', and difference-making evidence may help in singling out similarities and dissimilarities across different classes of individuals. Therefore, the heavier weight given to difference-making or mechanistic evidence has to do with the specific research questions at hand, not with alleged conceptual superiority of one type of evidence over another.

Second, biological and genetic factors are not enough to explain obesity. Biological factors alone, including genes playing specific roles in the mechanisms underlying obesity, do not wholly explain the rise of obesity because it has happened too quickly in evolutionary terms. Obesity, instead, is arguably the result of interactions between biology and environment. This thesis is supported, for instance, by Power and Schulkin (2009). Notably, Power and Schulkin (2009, p.5) hold that "much of the increase in human obesity is due to a mismatch between adaptive biological characteristics of our species and the modern environment, which has changed dramatically from the one under which we evolved".

What is put forward is then an ecological view of regulation of food intake: beside biological and genetic causes, there are also socio-economic causes of obesity. Before, life was hard and food scarce. Improvements of general living conditions, including access to food, resulted in more elevated intakes of food, especially of fats. The switch from agricultural economies to ones based on manufacturing also meant reduced costs of food and less calories to consume; also, exercising stops being part of normal working time and is confined to leisure time. These socio-economic changes explain the cross-sectional and timeseries patterns of obesity better than biological factors, addiction, and cultural changes (Philipson and Posner, 2008). This is again an example showing that the available mechanistic evidence supporting the hypothesis of a 'biological' increase in obesity is deficient.

Third, the question still looms as to why mechanistic evidence is needed after all, given that policy makers almost exclusively use information coming from risks. The answer to this question is that the more detailed the knowledge of the pathways, the more accurate the decision on what factors to intervene upon. Public health can hardly intervene on the genetic causes of obesity. Instead, significant results can be achieved if the patterns of behaviours of different types of obese people are identified. The MEND programme is again a good example. They aim to reduce child obesity by targeting families and parents. The key is to make parents realise that being overweight can cause their children not only health problems now and in the future, but also unhappiness, lack of confidence, depression. Thus, MEND appeals to psychological mechanisms (both in obese children at risk of depression and in the parents worried to avoid such situation) in order to improve health conditions.

Finally, the thesis that difference-making and mechanistic evidence are typically needed for causal assessment has the nice consequence that they usually 
help and support each other. This somehow goes against traditional views according to which to establish a causal claim different and independent sources of evidence are needed in order to triangulate. But the 'tangle' of differencemaking and mechanistic evidence on the one hand, and of bio-genetic and socioeconomic factors on the other hand exactly mirrors the complexity of disease causation (on this point, see especially Russo (2011)). Again, the phenomenon of obesity is exemplar in this respect.

\section{Conclusion}

Public health aims to preventing disease, prolonging life and promoting health of populations. Evidence-based public health, more specifically, seeks to reach such goals by means of population-level interventions that are decided on the basis of the best scientific evidence. Nevertheless, despite all this emphasis on evidence, what evidence ought to inform public health policy has not received a satisfactory answer yet. Studies on obesity are paradigmatic as they show the complexity of the issues behind public health policy and therefore help us drawing useful lessons about what evidence is needed.

In a nutshell, I defended the idea that public health policies ought to be informed by two types of evidence, broadly conceived: difference-making and mechanistic. The argument run in three steps. First, public health policy strongly relies on findings coming from epidemiology because it provides a population-level perspective on disease causation. Drawing on other work, I concluded that causal claims in epidemiology (and in medicine more generally) are established on the basis of difference-making and mechanistic evidence. Second, evidence-based public health heavily relies on evidence assessment but, I argued, this does not fully answer the question of what evidence backs up public health policy. Third, I provocatively pleaded for causally-based public health on the grounds that difference-making evidence supports considerations about 'what works for whom and in what circumstances' and that mechanistic evidence indicates where to intervene in the identified causal pathways.

One objection is that it is common practice to set up public health policies only on the basis of risks, that is on the basis of difference-making evidence. Far from urging that action is taken only with full mechanistic knowledge of the disease, the argument is that better policies can be envisaged if mechanistic evidence is explicitly taken into account, whenever available.

The advantage of adopting such a view is to have a coherent account where causal assessment, based on difference-making and mechanistic evidence, contributes to understanding disease and to take action to reduce its burden in population.

Acknowledgments I wish to thank Mike Joffe and Paolo Vineis for drawing my attention to obesity as an interesting case study. Rafael Blanc Moya, Lorenzo Casini, Brendan Clarke, Donald Gillies, Phyllis McKay Illari, Jon Williamson, have been very helpful in providing stimulating comments, wise suggestions, and bibliographical references. I wish also to thank the participants of the workshop 'Multi-level causation' held at the IHPST (Paris I) on 25-26 March 2010. 


\section{References}

Bechtel, W. and Abrahamsen, A. (2005). Explanation: a mechanist alternative. Studies in the History and Philosophy of the Biological and Biomedical Sciences, 36:421-441.

Bophal, R. (1997). Which book? a comparative review of 25 introductory epidemiology textbooks. Journal of Epidemiology and Community Health, 51:612-622.

Bophal, R. (1999). Paradigms in epidemiology textbooks: in the footsteps of thomas khun. American Journal of Public Health, 89(8):1162-1165.

Brownson, R. C., andT. L. Leet, E. A. B., and Gillespie, K. N. (2003). Evidence-Based Public Health. Oxford University Press, New York.

Brownson, R. C., Gurney, J. G., and Land, G. H. (1999). Evidence-based decision making in public health. Journal of Public Health Management Practice, 5(5):8697 .

Cartwright, N. (2008). Evidence for evidenced-based policy. Talk at a Home Office Seminar on Criminology and Evidence-Based Policy.

Cartwright, N. (2009). Causality, invariance, and policy. In Kincaid, H. and Ross, D., editors, The Oxford Handbook of Philosophy of Economics, chapter 15, pages 410-421. Oxford University Press.

Cartwright, N. (2010). Predicting 'it will work for us': (way) beyond statistics. In McKay-Illari, P., Russo, F., and Williamson, J., editors, Causality in the sciences, pages -. Oxford University Press.

Constantin, A., Costache, G., Sima, A. V., Glavce, C. S., Vladica, M., and Popov, D. L. (2010). Leptin g-2548a and leptin receptor q223r gene polymorphisms are not associated with obesity in romanian subjects. Biochemical and Biophysical Research Communications, 391(1):282-286.

Galea, S., Riddle, M., and Kaplan, G. A. (2010). Causal thinking and complex system approaches in epidemiology. International Journal of Epidemiology, 39:97-106.

Gillies, D. A. (2010). The Russo-Williamson thesis and the question of whether smoking causes heart disease. In McKay Illari, P., Russo, F., and Williamson, J., editors, Causality in the Sciences. Oxford University Press, Oxford.

Glasziou, P., Chalmers, I., Rawlins, M., and McCulloch, P. (2007). When are randomised trials unnecessary? picking signal from noise. British Medical Journal, 334:349-351.

Greener, J., Douglas, F., and van Teijlingen, E. (2010). More of the same? conflicting perspectives of obesity causation and intervention amongst overweight people, health professionals and policy makers. Social Science $\&$ Medicine, 70:10421049.

Guyatt, G. and Drummond, R. (2002). Users Guides to the Medical Literature: A Manual for Evidence-Based Practice. American Medical Association, Chicago.

Illari, P. M. (2010). Mechanistic evidence: Disambiguating the Russo-Williamson thesis.

Killoran, A. and Kelly, M. P., editors (2010). Evidence-based public health. Effectiveness and efficiency. Oxford University Press, New York. 
Mouchart, M. and Russo, F. (2010). Causal explanation: recursive decompositions and mechanisms. In McKay-Illari, P., Russo, F., and Williamson, J., editors, Causality in the sciences. Oxford University Press.

Philipson, T. and Posner, R. (2008). Is the obesity epidemic a public health problem? a decade of research on the economics of obesity. Technical Report Working paper 14010, The National Bureau of Economic Research.

Porta, M., editor (2008). A dictionary of epidemiology. Oxford University Press, fifth edition.

Power, M. and Schulkin, J. (2009). The evolution of obesity. The John Hopkins University Press, Baltimore.

Russo, F. (2009). Variational causal claims in epidemiology. Perspectives in Biology and Medicine, 52(4):540-554.

Russo, F. (2011). Causal webs in epidemiology. Paradigmi, (In Press).

Russo, F. and Williamson, J. (2007). Interpreting causality in the health sciences. International Studies in Philosophy of Science, 21(2):157-170.

Russo, F. and Williamson, J. (2011). Generic vs. single-case causal knowledge. the case of autopsy. European Journal for Philosophy of Science.

Rychetnik, L., Hawe, P., Waters, E., Barratt, A., and Frommer, M. (2004). A glossary for evidence based public health. Journal of Epidemiology and Community Health, 58:538-545.

Salmon, W. (1984). Scientific Explanation and the Causal Structure of the World. Princeton University Press, Princeton.

Steel, D. (2008). Across the boundaries. Extrapolation in biology and social science. Oxford University Press. 